\title{
Synthesis of Bipyridyl-Branched Polyoxazoline and Its Gelation by Means of Metal Coordination
}

\author{
Yoshiki Chujo, ${ }^{*}$ Kazuki SAda, ${ }^{* *}$ and Takeo SAEgusA ${ }^{* * *}$ \\ Department of Synthetic Chemistry, Faculty of Engineering, Kyoto University, \\ Yoshida, Sakyo-ku, Kyoto 606-01, Japan
}

(Received November 4, 1992)

\begin{abstract}
Polyoxazoline hydrogel was prepared through the coordination of the metal ions to 2,2'-bipyridyl-branched poly( $N$-acetylethylenimine) (PAEI), which was synthesized from the partially hydrolyzed PAEI by the reaction with $3-\left\{4-\left(4^{\prime}\right.\right.$-methyl-2,2'-bipyridyl $\left.)\right\}$ propanoic acid in the presence of dicyclohexylcarbodiimide. Concentrated solution of 2,2'-bipyridyl-branched PAEI gave polyoxazoline gel in a good yield by the treatment with metal salts such as iron(II) sulfate, or ruthenium(III) trichloride. The resulting colored gel was highly swollen in water and stable at ambient temperature for handling. In the case of nickel(II) or cobalt(II) ions, the formed intermolecular cross-linking points via the coordination (gel state) were labile due to the rapid ligand exchange reaction of the cross-linking from intermolecular to intramolecular manner. The nickel(II) and cobalt(II) gels became soluble in a large amount of water within a few hours. A series of PAEIs having varying amounts of the functional groups were prepared and subjected to the cross-linking reaction by the coordination with iron(II). The swelling degree and the stability of the gel in water depended on the content of the functional groups in the prepolymers. Water uptake was up to 56 multiples of its own weight in dry state.
\end{abstract}

KEY WORDS Ring-Opening Polymerization / Polyoxazoline / Bipyridyl /

Metal Coordination / Cross-Linking / Hydrogel / Water Swelling Property /

Cross-linking through the interaction between metal ions and polymeric ligands has been of interest for the preparation of networks. Most of these investigations focused on their physical properties in the concentrated solution. The complex formation of the metal ion with the polymer bearing the ligand groups was first claimed by Kuhn and his coworkers. ${ }^{1}$ They discussed on the network formation from poly(vinyl alcohol) fiber by the treatment with $\mathrm{Cu}(\mathrm{II})$ salt with respect to the rheological measurement.

Polymers containing carboxylic acids or hydroxyl groups such as poly(acrylic acid), ${ }^{2}$ poly $\{$ bis(carboxylatophenoxy)phosphazene $\},{ }^{3}$ polysaccharide, ${ }^{4-6}$ and cellulose derivatives ${ }^{7}$ are known to form stable hydrogels by the treatment with metal salts under the specific conditions $(\mathrm{pH}$, concentration, ionic strength, etc.). A large number of ions (Ca(II), ${ }^{3}$ $\mathrm{Cu}(\mathrm{II}),{ }^{2,3} \mathrm{Al}(\mathrm{III}),{ }^{3,7} \mathrm{Cr}(\mathrm{III}),{ }^{8} \mathrm{Ti}(\mathrm{III}),{ }^{6,7}$ and borate $\left.^{4,5}\right)$ are used as a cross-linking agent. These gels have been used widely in a field of industrial chemistry. However, the mechanism of the cross-linking has not been fully established. The stability and the physical properties often depend on their specific conditions for their preparations. In these systems, it is difficult to control the swelling properties and to reveal the nature of the

* To whom all correspondence should be addressed.

** Present address: Department of Chemistry, Faculty of Engineering, Gifu University, Yanagido, Gifu 501-11, Japan.

*** Present address: KRI International Inc., Chudoji, Minamimachi, Shimogyo-ku, Kyoto 600, Japan. 
cross-linking.

On the other hand, the polymeric ligands such as hydroxamic acid, ${ }^{9}$ salicylic acid, ${ }^{10}$ pyridine, ${ }^{11}$ iminodiacetic acid, ${ }^{12}$ acetylacetone, ${ }^{13,14}$ and crown ether ${ }^{15}$ were prepared previously. Some of them were reported as chelate resins from a viewpoint of ion separation or ion extraction engineering. Nevertheless, the use of these polymer ligands for the cross-linking formation has been less known. Eichinger and his coworkers showed in a series of their studies ${ }^{13,14,16}$ that the elastomer containing acetylacetone groups gave the stable gel by the treatment with metal ions (Cr(III)) in an organic solvent. In the case of a watersoluble polymer, Winston et al. reported the preparation and the gelation of the hydroxamic acid derivated poly(acrylamide). ${ }^{9}$ We wish to report here the preparation of the PAEI hydrogel through the coordination between the pendant ligand group and metal ions.

Hydrogels are known to be one of the most interesting polymeric materials and used in the various fields such as household articles, horticulture, coating materials, and civil engineering for these several years. The commercially available polymeric hydrogels are usually based on the cross-linked polyelectrolytes such as poly(acrylic acid) salt or poly(styrene sulfonate). A large volume-change of the gels with temperature or with solvent has successfully been explained by the phase transition of the cross-linked ionic gel. The generality of these phenomena has been investigated extensively by Tanaka's group. ${ }^{17}$ In aqueous salts, the swelling degrees of these ionic hydrogels are known to be diminished largely in comparison with those of non-ionic hydrogels made from poly(oxyethylene) ${ }^{18}$ or poly(acrylamide). ${ }^{19}$

We have been studying the ring-opening polymerization of 2-methyl-2-oxazoline for these several years. ${ }^{20}$ The resulting $\operatorname{poly}(\mathrm{N}$ acetylethylenimine) (PAEI) (polyoxazoline) has high hydrophilicity and good compatibility with several commodity polymers. ${ }^{21}$ We have reported the preparation of PAEI gels by two independent methods, partially hydrolysis followed by cross-linking ${ }^{22}$ and copolymerization. ${ }^{23}$ They have characteristic properties as a non-ionic hydrogel, i.e., a large swelling degree both in water and in aqueous salts. Recently, we also described the photo-reversible hydrogel system ${ }^{24}$ by the photo-coupling reaction of coumarin-modified PAEI and the thermally-reversible hydrogel system ${ }^{25}$ based on this polymer by means of Diels-Alder reaction.

In this article, we describe a novel method for the preparation of PAEI hydrogels through the coordination between 2,2'-bipyridylbranched PAEI and metal ions. 2,2'-Bipyridyl derivatives are known to be the strongest ligands for transition metal ions such as $\mathrm{Fe}(\mathrm{II}), \mathrm{Ni}(\mathrm{II}), \mathrm{Cu}(\mathrm{II}), \mathrm{Ru}(\mathrm{II})$, and $\mathrm{Os}(\mathrm{III})$ to form the stable complexes. ${ }^{26}$ These complexes have been studied extensively as photo-sensitizers $^{27,28}$ or as synthetic building blocks for supramolecular assemblies. ${ }^{29}$ The ruthenium(II) tris complex is especially well-known for the photo-dissociation of water, and is of interest in the solar energy conversion program. ${ }^{28}$ The polymeric 2,2'-bipyridyl ligands were also prepared, however, most reports focused on the chemistry of the photo-excited ruthenium complex. ${ }^{30}$ Only a few example showed the coordination between bipyridyl moiety in the polymer and transition metal ions. $^{31}$

\section{EXPERIMENTAL}

\section{General Procedure}

UV spectra were measured on a Hitachi 200 UV-VIS spectro-photometer. IR spectra were recorded on a Hitachi 260-50 grating spectro-photometer. ${ }^{1} \mathrm{H}$ NMR spectra were obtained on a Hitachi R-600 $(60 \mathrm{MHz})$, or on a JEOL JNM-JX-400 (400 MHz) spectrometer. All ${ }^{1} \mathrm{H}$ NMR spectra were recorded in $\mathrm{CDCl}_{3}$ solutions with tetramethylsilane inter- 
nal standard. GPC analysis was carried out on a Tosoh CCPD (TSK gel G4000) after calibration with the standard polystyrene samples.

\section{Materials}

All solvents and reagents were used as supplied except the following materials. $N, N$ Dimethylformamide (DMF) was distilled from barium oxide and then from calcium hydride under reduced pressure. Nitromethane was distilled from phosphorus pentoxide under nitrogen. Acetonitrile was distilled from calcium hydride and then distilled from phosphorus pentoxide under nitrogen. Methyl $p$-toluenesulfonate was distilled under reduced pressure. 2-Methyl-2-oxazoline or diethylamine was distilled from potassium hydroxide. Tetrahydrofuran (THF) and triethylamine were distilled from lithium aluminum hydride under nitrogen. Ethyl bromoacetate was distilled under reduced pressure. Ethyl chloroformate was distilled under nitrogen atmosphere. According to the previous method, poly $(N$-acetylethylenimine) (PAEI) was prepared by the ring-opening polymerization of 2-methyl-2-oxazoline in the presence of methyl $p$-toluenesulfonate as an initiator, and was partially hydrolyzed by the treatment with aqueous sodium hydroxide. ${ }^{22}$

3-\{4-(4'-Methyl-2,2'-bipyridyl) $\}$ propanoic Acid

To a pre-cooled $\left(-78^{\circ} \mathrm{C}\right)$ mixture of $1.99 \mathrm{~N}$ $n$-hexane solution $(11.1 \mathrm{ml}, 22.1 \mathrm{mmol})$ of lithium diisopropylamide (LDA) in $20 \mathrm{ml}$ of dry THF was added a solution of $3.70 \mathrm{~g}$ (20.1 mmol) of 4,4'-dimethyl-2,2'-bipyridyl (1) in $190 \mathrm{ml}$ of dry THF for $30 \mathrm{~min}^{32}$ The resulting dark brown solution was stirred for $2 \mathrm{~h}$ at $-78^{\circ} \mathrm{C}$. This lithiated bipyridyl solution was added dropwise to a solution of $3.79 \mathrm{~g}$ $(22.7 \mathrm{mmol})$ of ethyl bromoacetate in $40 \mathrm{ml}$ of dry THF at the same temperature using Teflon cannular. The yellow solution was allowed to warm to room temperature and stirred overnight. After the insoluble part was removed by filtration, the red-orange solution was concentrated under reduced pressure. The residue was extracted with dichloromethane, and the solution was passed through an alumina column (eluent; dichloromethane). The solvent was removed in vacuo to yield bipyridyl ester (2) as a pale yellow oil (3.12 g, $57 \%$ ), which was used in the next step without further purification. ${ }^{1} \mathrm{H}$ NMR; $\delta 1.23(\mathrm{t}, 3 \mathrm{H}$, $J=7 \mathrm{~Hz}), 2.43$ (s, 3H), 2.70-3.04 (m, 4H), 4.14 $(\mathrm{q}, 2 \mathrm{H}, J=7 \mathrm{~Hz}), 7.16(\mathrm{t}, 2 \mathrm{H}), 8.26(\mathrm{~s}, 2 \mathrm{H})$, $8.44(\mathrm{~m}, 2 \mathrm{H})$.

The ethyl ester (2) was dissolved in $c a .50 \mathrm{ml}$ of THF. To this solution was added $20 \mathrm{ml}$ of aqueous sodium hydroxide $(2.77 \mathrm{~g}, 61 \mathrm{mmol})$. After stirring overnight at room temperature, the solvent was removed under reduced pressure. The white residue was redissolved in distilled water $(c a .20 \mathrm{ml})$. The solution was extracted with two portions of ethyl acetate and the aqueous layer was acidified carefully with concentrated sulfuric acid. The resulting white precipitate was collected and washed with chilled distilled water. The carboxylic acid (3) was dried in vacuo to yield $0.909 \mathrm{~g}$ $(53 \%) .{ }^{1} \mathrm{H}$ NMR (DMSO- $\left.d_{6}-\mathrm{CDCl}_{3}\right) ; \delta 2.43$ (s, 3H), 2.70-3.04 (m, 4H), $7.18(\mathrm{t}, 2 \mathrm{H}), 8.19$ $(\mathrm{s}, 2 \mathrm{H}), 8.49$ (d, 2H), IR (KBr); 3380, 3050, $2900,1700,1600,1550,1460,1415,1200$, $1005,895,830 \mathrm{~cm}^{-1}, \mathrm{MS} ; 242\left(\mathrm{M}^{+}\right), 197$ $\left(\mathrm{M}^{+}-\mathrm{COOH}\right), \mathrm{UV}$ (methanol); $\lambda_{\max }=289 \mathrm{~nm}$ $(\varepsilon=15000), 239 \mathrm{~nm} \quad(\varepsilon=13000), \mathrm{mp}=190$ $192^{\circ} \mathrm{C}$

\section{$N, N$-Diethyl 3-\{4-(4'-Methyl-2,2'-bipyridyl $)\}-$ propionamide (4)}

To a pre-cooled solution $\left(-15^{\circ} \mathrm{C}\right)$ of $3-\{4-$ (4'-methyl-2,2'-bipyridyl) $\}$ propanoic acid (1) $(0.538 \mathrm{~g}, 2.24 \mathrm{mmol})$ in DMF $(40 \mathrm{ml})$ was added dropwise $0.470 \mathrm{~g}(4.65 \mathrm{mmol})$ of triethylamine and $0.325 \mathrm{~g}(2.99 \mathrm{mmol})$ of ethyl chloroformate while maintaining the temperature at $-15^{\circ} \mathrm{C}$. After additional stirring for $5 \mathrm{~min}$ at $-15^{\circ} \mathrm{C}, 0.455 \mathrm{~g}(6.22 \mathrm{mmol})$ of diethylamine was added dropwise to this 
solution. The resulting solution was allowed to warm to room temperature overnight with stirring. After the white solid was removed, DMF was evaporated under reduced pressure. The residue was extracted with $30 \mathrm{ml}$ of dichloromethane and washed with $5 \%$ aqueous sodium carbonate $(10 \mathrm{ml})$ and then twice with distilled water $(10 \mathrm{ml})$. The aqueous layer was extracted with one portion of dichloromethane $(20 \mathrm{ml})$. The organic layers were combined and dried over anhydrous magnesium sulfate. After the solution was concentrated under reduced pressure, the brown oil was passed through an alumina column (eluent; ethyl acetate). After drying in vacuo, $0.343 \mathrm{~g}(52 \%)$ of $N, N$-diethyl amide derivative (4) was obtained as a colorless oil. ${ }^{1} \mathrm{H}$ NMR; $\delta 1.07(\mathrm{t}, \quad 6 \mathrm{H}, \quad J=7 \mathrm{~Hz}), 2.39$ $(\mathrm{s}, 3 \mathrm{H}), 2.71-3.07(\mathrm{~m}, 4 \mathrm{H}), 3.36(\mathrm{q}, 4 \mathrm{H}$, $J=7 \mathrm{~Hz}), 7.16(\mathrm{t}, 2 \mathrm{H}), 8.26(\mathrm{~s}, 2 \mathrm{H}), 8.52(\mathrm{~m}$, 2H), IR (neat); 3050, 2980, 2930, 2900, 1635, $1600,1550,1460,1415,1380,1260,1210,995$, $820,800 \mathrm{~cm}^{-1}, \mathrm{MS} ; 297\left(\mathrm{M}^{+}\right), 225\left(\mathrm{M}^{+}-\right.$ $\left.\mathrm{NEt}_{2}\right), 197\left(\mathrm{M}^{+}-\mathrm{CONEt}_{2}\right)$, UV (methanol); $\lambda_{\text {max }}=282 \mathrm{~nm}(\varepsilon=15500)$.

Poly( $N$-acetylethylenimine $)-p o l y\left(N-\left[3-\left\{4-\left(4^{\prime}-\right.\right.\right.\right.$ methyl-2,2'-bipyridyl)\}propanoyl]ethylenimine) Copolymer (8)

A typical procedure is as follows. A benzene $(5 \mathrm{ml})$ suspension of a mixture of $0.395 \mathrm{~g}$ of $8.8 \%$-hydrolyzed PAEI (7) $(0.411 \mathrm{mmol} \mathrm{NH}$ equivalent $)$ and $0.183 \mathrm{~g}(0.756 \mathrm{mmol})$ of $3-\{4-$ (4'-methyl-2,2'-bipyridyl)\}propanoic acid (3) was freeze-dried. After introducing dry nitrogen, the mixture was dissolved in $5 \mathrm{ml}$ of dry acetonitrile and $3 \mathrm{ml}$ of freshly distilled DMF under nitrogen, and cooled to $0^{\circ} \mathrm{C}$. To this solution, $0.245 \mathrm{~g}(1.19 \mathrm{mmol})$ of dicyclohexylcarbodiimide (DCC) was added. After stirring for 3 days at room temperature, the resulting dicyclohexylurea was removed by filtration, and the filtrate was concentrated to yield a pale orange polymer, which was redissolved in methanol and reprecipitated into diethyl ether. Bipyridyl-branched PAEI (8) was ob- tained after freeze-drying with benzene. Yield was $347 \mathrm{mg}(71 \%)$.

\section{Gelation by $\mathrm{Fe}(\mathrm{II})$}

A typical gelation of bipyridyl-branced PAEI (8) is as follows. 8 (8.8\%-functionalized, $50 \mathrm{mg}, 0.042 \mathrm{mmol}$ equivalent) was dissolved in $0.1 \mathrm{ml}$ of distilled water. Under vigorous stirring, $40 \mathrm{ml}(0.03 \mathrm{mmol})$ of the stock solution $(2.085 \mathrm{~g} / 10 \mathrm{ml})$ of aqueous ferrous sulfate heptahydrate was added to this polymer solution. The red gel (9a) obtained was stirred for additional $10 \mathrm{~min}$ and was immersed in $10 \mathrm{ml}$ of methanol for $30 \mathrm{~min}$. The resulting gel was collected by filtration and washed with methanol. After drying in vacuo, $36.9 \mathrm{mg}$ $(70 \%)$ of the red PAEI gel (9a) was obtained.

\section{Gelation by Aqueous Metal Salts}

Gelation reactions of bipyridyl-branched PAEI (8) by other metal salts were carried out in a similar fashion. In the case of ruthenium trichloride, the ratio of bipyridyl moiety to the metal salt was adjusted at three and the reaction mixture was heated at $100^{\circ} \mathrm{C}$ for 3 days in a sealed tube. After washing with methanol, the orange-yellow gel was collected by filtration and dried in vacuo.

\section{Swelling Properties}

The equilibrium swelling properties in water were measured by the method described in earlier papers. ${ }^{22-25}$

\section{RESULTS AND DISCUSSION}

\section{Synthesis and Characterization of Bipyridyl- Branched PAEI (8)}

The preparation of various bipyridyl derivatives is represented in Scheme 1. Commercially available 4,4'-dimethyl-2,2'-bipyridyl (1) was employed as a starting material. ${ }^{32}$ The bipyridyl group was introduced to PAEI by the reaction of partially hydrolyzed polymer (7) with 3-\{4-(4'-methyl-2,2'-bipyridyl)\}propanoic acid (3) as shown in Scheme 2. N,N-Diethyl- 
amide (4) was prepared as a model compound of the bipyridyl-carrying unit of PAEI. A series of the partially hydrolyzed PAEI samples (7) with various degrees of hydrolysis were prepared by the ring-opening polymerization of 2-methyl-2-oxazoline (5) followed by alkaline hydrolysis according to an earlier procedure. ${ }^{22}$ Two kinds of the partially hydrolyzed PAEIs (7) $\left(M_{n}=15800\right.$ and 25300) were used for the following reactions.

From the results of UV spectrum of the bipyridyl-polymer (8) (3.8\% functionality) in methanol, the absorption maximum of the bipyridyl group was found at $284 \mathrm{~nm}$. A model compound, $N, N$-diethyl derivative (4), has a similar absorption, $\lambda_{\max }=282 \mathrm{~nm}(\varepsilon=$ 15500). Thus, the degree of substitution of bipyridyl group in 8 could be estimated on the basis of the absorption coefficient for the model compound (4).

In Figure 1 is reported the ${ }^{1} \mathrm{H}$ NMR spectrum of bipyridyl-branched PAEI (8). The peak of methylene protons adjacent to the secondary amino group $(\delta 2.8)$ has disappeared, while the pyridine protons $(\delta 7.2-8.6)$ and the singlet of methyl protons $(\delta 2.45)$ of methyl-bipyridyl group are observed. The degree of substitution of the ligand could be calculated from the integral ratio of the bipyridyl protons and the remaining $N$-acetyl protons ( $\delta$ 2.1).

Table I summarizes the results of the preparation of bipyridyl-containing PAEI (8) together with the degrees of substitution. The degrees of substitution estimated from UV spectra were found to be close to those from ${ }^{1} \mathrm{H}$ NMR. These values also corresponded well to the degrees of hydrolysis of the starting partially hydrolyzed PAEIs. This result confirms the completion of the condensation reaction for the preparation of $\mathbf{8}$. The swelling properties of the gels can be controlled by the degree of substitution (vide infra), that is, by the degree of hydrolysis in 7.

From the GPC analysis of the polymers, no significant change of the molecular weight was
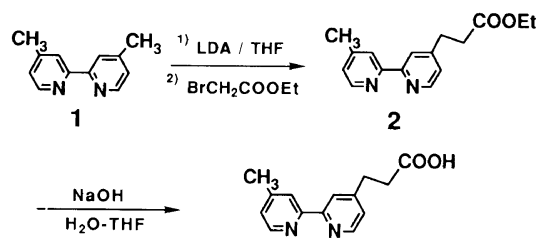

3

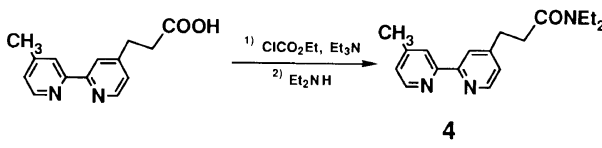

Scheme 1.
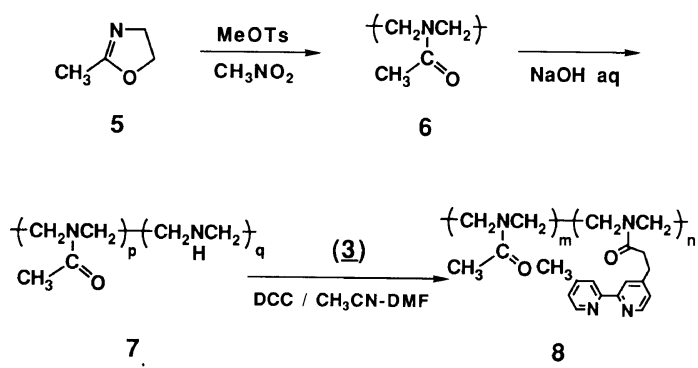

Scheme 2.

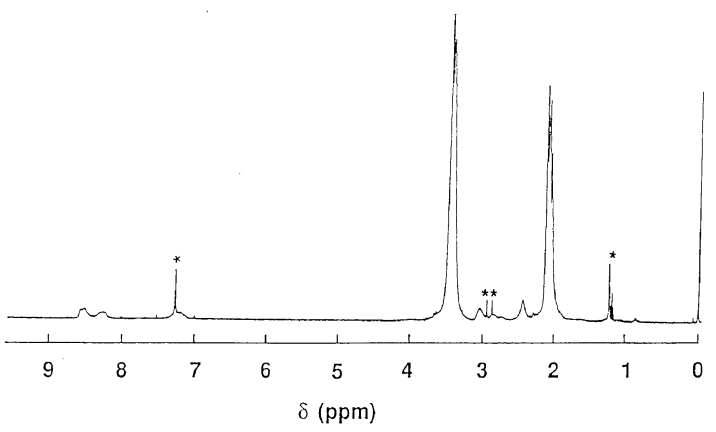

Figure 1. ${ }^{1} \mathrm{H}$ NMR (400 MHz) spectrum of 2,2'-bipyridyl-branched (8.8\%) PAEI (8).

observed in comparison with that of the starting polymer (7). The molecular weight distribution $\left(M_{n}=28200, M_{w}=43800, M_{w} / M_{n}=1.5\right)$ of 8 was also similar to that of 7 . The GPC trace detected by UV at $284 \mathrm{~nm}$ demonstrated that the bipyridyl group was introduced onto the PAEI. These data indicate that no significant chain degradation or cross-linking occurred during the condensation step for the 
Table I. Preparation of bipyridyl-branched PAEI (8)

\begin{tabular}{|c|c|c|c|c|c|}
\hline \multirow{2}{*}{ Run } & \multicolumn{2}{|c|}{ (7) } & \multirow{2}{*}{$\begin{array}{c}(\mathbf{8}) \\
\text { Yield } / \%\end{array}$} & \multicolumn{2}{|c|}{$n /(m+n)$} \\
\hline & $\bar{M}_{n}^{\mathrm{a}}$ & $q /(p+q)^{\mathrm{b}}$ & & $\mathrm{UV}^{\mathrm{c}}$ & ${ }^{1} \mathrm{H} \mathrm{NMR}^{\mathrm{d}}$ \\
\hline 1 & 25300 & 0.022 & 83 & 0.010 & \\
\hline 2 & 25300 & 0.041 & 85 & 0.033 & \\
\hline 3 & 25300 & 0.085 & 71 & 0.081 & 0.088 \\
\hline 4 & 25300 & 0.169 & 67 & 0.144 & \\
\hline 5 & 15800 & 0.051 & 92 & 0.038 & \\
\hline
\end{tabular}

a Determined by GPC (PSt standard).

b Calculated by titration.

${ }^{c}$ Determined by UV at $\lambda_{\max }=284 \mathrm{~nm}\left(\varepsilon=1.55 \times 10^{4}\right)$.

${ }^{d}$ Determined by ${ }^{1} \mathrm{H}$ NMR (Acetyl protons $v s$. ring protons).

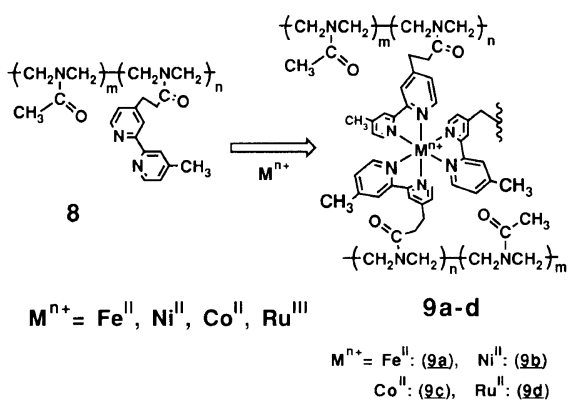

Scheme 3.

preparation of these functionalized PAEIs. The GPC also showed that there was no free ligand contamination in $\mathbf{8}$.

Gelation of Bipyridyl-Branched PAEIs (8) by $\mathrm{Fe}(\mathrm{II})$ Ion and the Swelling Properties of the Gels in Water

Dilute solutions gave no gelation on treatment with metal ions. The gelation reaction through metal coordination was carried out in a relatively concentrated solution $(c a .1 / 3$, $\mathrm{w} / \mathrm{v}$ ) of bipyridyl-branched PAEI (8) with aqueous metal salts (Scheme 3 ). On addition of a few drops of aqueous ferrous sulfate, the polymer solution turned red and simultaneously a gel (9) was formed, which was stable for handling, and was highly swollen in water.

Table II shows the results of $\mathrm{Fe}(\mathrm{II})$-induced gelation of bipyridyl-branched PAEIs (8) with a variety of degrees of substitution and the
Table II. Gelation of Bpy-PAEI (8) by Fe(II) ion

\begin{tabular}{|c|c|c|c|c|}
\hline \multirow{2}{*}{$\operatorname{Run}^{a}$} & \multicolumn{2}{|c|}{ (8) } & \multicolumn{2}{|c|}{ (9a) } \\
\hline & $\bar{M}_{n}^{\mathrm{b}}$ & $n /(m+n)^{\mathbf{c}}$ & Yield/\% & $\begin{array}{l}\text { Swelling } \\
\text { degree }^{\mathrm{d}}\end{array}$ \\
\hline 1 & 28200 & 0.010 & $0^{\mathbf{e}}$ & - \\
\hline 2 & 28200 & 0.033 & 90 & 56 \\
\hline 3 & 28200 & 0.081 & 78 & 11 \\
\hline 4 & 28200 & 0.144 & 72 & 4.7 \\
\hline 5 & 15800 & 0.038 & 83 & 18 \\
\hline \multicolumn{5}{|c|}{$\begin{array}{l}\text { a }[\mathrm{Fe}(\mathrm{II})] /[\mathrm{Bpy}]=2 / 3 \text { (polymer } 50 \mathrm{mg} \text {, water } c a .100 \mu \mathrm{l}) \\
\text { b } \\
\text { Determined by GPC (PSt standard). } \\
\text { c } \text { Determined by UV. } \\
\left.\text { d g- } \mathrm{H}_{2} \mathrm{O} / \mathrm{g} \text {-dry gel (in water for } 24 \mathrm{~h}\right) . \\
\text { e } \mathrm{No} \text { gelation was observed. }\end{array}$} \\
\hline
\end{tabular}

swelling properties of the resulting gels. In all cases except Run 1, the PAEI gel (9) was obtained in a good yield. No gelation was observed in the absence of pendant ligands in the polymer, i.e., in the cases of hydrolyzed PAEI (7) and of non-hydrolyzed parent polymer (6). These results indicate that the intermolecular cross-linking by coodination of the bipyridyl moieties in $\mathbf{8}$ to metal ion caused the formation of stable hydrogel. Even when the degree of substitution of bipyridyl groups was relatively small $(1.0 \%$, Run 1$)$, the complexation between the ligands in the polymer and $\mathrm{Fe}$ (II) ion was proved to occur from the results of UV spectra. However, no gel was formed in this case, even in much higher 

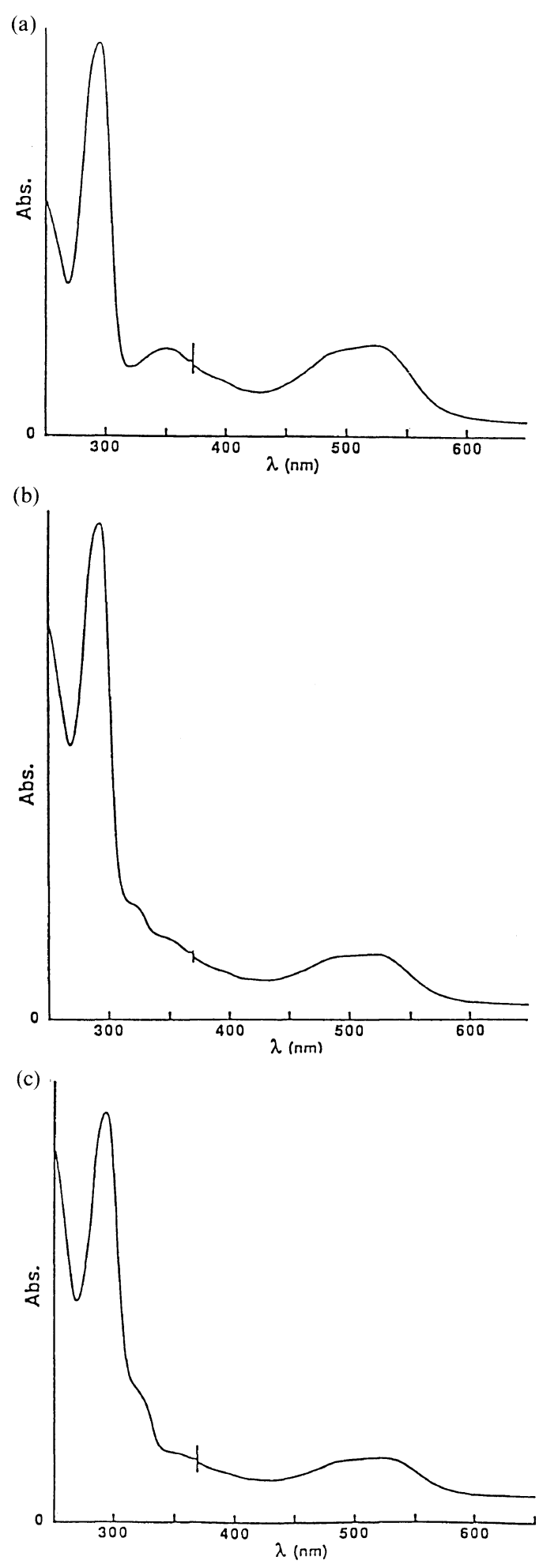

Table III. Gelation of Bpy-PAEI (8) by various metal ions

\begin{tabular}{|c|c|c|c|c|}
\hline \multirow{2}{*}{$\operatorname{Run}^{\mathrm{a}}$} & \multirow{2}{*}{$\begin{array}{l}\text { Metal } \\
\text { salt }\end{array}$} & \multicolumn{3}{|c|}{$(9 a-d)$} \\
\hline & & Yield/\% & $\begin{array}{l}\text { Swelling } \\
\text { degree }^{b}\end{array}$ & Color \\
\hline 1 & $\mathrm{FeSO}_{4}$ & 83 & 18 & Red \\
\hline 2 & $\mathrm{NiCl}_{2}$ & 78 & $-^{c}$ & Colorless \\
\hline 3 & $\mathrm{CoCl}_{2}$ & $0^{\mathrm{d}}$ & - & Orange-yellow \\
\hline $4^{e}$ & $\mathrm{RuCl}_{3}$ & 97 & 14 & Red-orange \\
\hline
\end{tabular}

a $\left[\mathrm{M}^{\mathrm{n}+}\right] /[\mathrm{bpy}]=2 / 3$ (polymer $50 \mathrm{mg}$, water ca. $100 \mu \mathrm{l}$ ). Prepolymer, $\overline{M n}=15800$ 2,2'-bipyridyl content $3.8 \%$.

b g- $\mathrm{H}_{2} \mathrm{O} / \mathrm{g}$-dry gel (in water for $24 \mathrm{~h}$ ).

c Dissolved within $30 \mathrm{~min}$.

d No gelation was observed.

e $[\mathrm{Ru}(\mathrm{II})] /[\mathrm{bpy}]=1 / 3,80^{\circ} \mathrm{C}, 3$ days.

concentration of the polymer. With increasing degrees of substitution of the bipyridylbranched PAEIs (8), the degree of swelling of 9a in water decreased. Thus, the average distance between the nearest two branches could be controlled by the content of the ligands in $\mathbf{8}$. Figure $2 \mathrm{a}$ shows the UV spectrum of the swollen gel film (9a). The absorption maxima are located at 278 and $517 \mathrm{~nm}$. These peaks are not only similar to those of the model complex prepared from the bipyridyl derivative (4) and $\mathrm{Fe}(\mathrm{II})$ ion (Figure $2 \mathrm{~b}$ ), but also to those of the polymer complex in dilute solution (Figure 2c).

\section{The Effect of Metal Ions on the Stability of the Gels}

Various metal salts (Fe(II), Ni(II), Co(II), $\mathrm{Ru}(\mathrm{III}))$ were employed for the gelation reaction through the coodination to the bipyridylbranched PAEIs (8) (Table III). The reaction conditions were almost the same as that of the ferrous complex. In the case of ferrous

Figure 2. UV absorption spectra of (a) the swollen gel film of iron(II) 2,2'-bipyridyl-branched (3.8\%) PAEI complex (9a) in methanol, (b) iron(II) complex of $N, N$ diethyl 3-\{4-(4'-methyl-2,2'-bipyridyl) $\}$ propionamide (4), and (c) iron(II) 2,2'-bipyridyl-branched (3.8\%) PAEI complex (9a) prepared in a dilute solution. 
sulfate discussed above, the resulting red gel (9a) was formed in a good yield, which had enough stability for handling and was swollen in water up to 56 times its own weight.

Treatment of bipyridyl-branched polymer (8) with an aqueous nickel(II) salt, which yields complexes with the greatest overall stability constant, ${ }^{33)}$ gave a colorless gel product (9b). However, this gel was not stable in water, and gradually dissolved within a few hours to give a water-soluble metal complex. Cobalt(II), one of the strongest binding cations for bipyridyl groups, ${ }^{33}$ also failed to form a stable gel in methanol, even in the highly concentrated state. In these two cases, the polymer networks $(\mathbf{9 b}, 9 \mathbf{9})$ were less stable in comparison with $\mathbf{9 a}$, and turned out to be soluble by immersing in a large amount of water. The UV spectra of the resulting solutions of $9 \mathbf{b}$ and $9 \mathbf{c}$ show definitely the formation of the complexes of the PAEI-bound bipyridyls with these metal cations $\left(\lambda_{\max }=\right.$ 296, $302 \mathrm{~nm}$ for $\mathbf{9 b}$, and $\lambda_{\max }=295,303 \mathrm{~nm}$ for $9 c)$. The formation constants ${ }^{33}$ of these model complexes (2,2'-bipyridyl complexes with nickel(II), cobalt(II), and iron(II)) indicate that they have sufficient thermodynamically stability to form tris-complexes under the experimental conditions. Though iron trisbipyridyl complex is not thermodynamically more stable than those of the other two metal ions, only iron(II) ion gave a stable hydrogel.

The rate of dissociation of the tris-chelate complexes of these metal ions was investigated by Basolo et al. ${ }^{34}$ From their results, the rate of ligand exchange decreases in the order: $\mathrm{Co}(\mathrm{II})>\mathrm{Ni}(\mathrm{II})>\mathrm{Fe}(\mathrm{II})$. This order is consistent with our results, that is, the order of stability of the polymer network of bipyridylbranched PAEI and metal ions (9a, 9b, 9c). The disappearance of intermolecular crosslinking was found to occur by ligand exchange reactions of the polymeric ligands (Scheme 4). The initial polymer network formation occurred by intermolecular croos-linking through the metal ion coordination in highly con- centrated solutions. If the rate of ligand exchange reaction of the polymeric ligands is fast, that is, the resulting complex is labile, the first formed intermolecular cross-linkages shift rapidly to intramolecular cross-linkages in a large amount of water. This change of the coordination bond from inter to intra leads to the disappearance of the polymer network without an apparent change in the concentration of the metal complex. Concerning the conversion from intermolecular complex (gel) to intramolecular one, Winston has pointed out in the case of hydroxamic acid copolymers and iron(III) ion. ${ }^{9}$ Thus, the interconversion from the gel to the polymer in $9 \mathbf{b}$ and $9 \mathbf{c}$, can be rationalized. On the other hand, $\mathrm{Fe}(\mathrm{II})$ and $\mathrm{Ru}(\mathrm{III})$ (vide infra) complexes (9a, 9d) are known to be so inert that the formed intermolecular networks are expected to disappear very slowly or do not at all. In our studies, the gels from $\mathrm{Fe}$ (II) salts were found to be stable by immersing in a large amount of water. Thus, the stability of the coordination gel prepared from these metal ions and 2,2'-bipyridyl-branched PAEIs was found to depend on their kinetic inertness, not on their thermodynamic stability.

The ruthenium complex (9d) was also formed by heating with ruthenium trichloride in water for 3 days to give an orange-red gel. The complexation required heating for 3 days because of the kinetically slow formation of ruthenium complex. ${ }^{30}$ However, once formed,

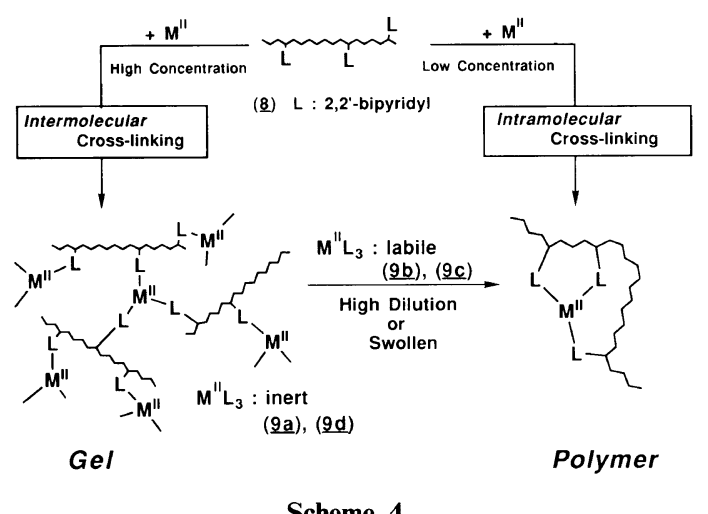

Scheme 4. 
the gel (9d) was sufficiently stable for handling and could be swollen in water. Compared with the iron complex (9a), 9d was extremely stable, i.e., the swelling degree did not change after the gel was kept immersed in water for more than 1 month at room temperature. This stability of 9d is taken to depend both on thermodynamic stability and kinetic inertness.

The degrees of swelling of the gels by coordination in water are shown in Table III. A value for the nickel(II) complex (9b) could not be measured because of the fast solubilization in water. On the other hand, iron(II) (9a) and ruthenium(III) (9d) complexes were stable and the swelling degrees could be measured without the apparent disappearance of the polymer networks by immersion in water for $24 \mathrm{~h}$. These values (about 15-18) were close to each other and were in good agreement with the results of the degrees of swelling of a series of the functionalized PAEIs ${ }^{35}$ (Table II).

\section{CONCLUSIONS}

The bipyridyl-branched PAEIs (8) were easily prepared from the partially hydrolyzed PAEIs (7) by the usual modification method. These functionalized PAEIs (8) were employed for reaction with metal ions such as $\mathrm{Fe}(\mathrm{II}), \mathrm{Ru}(\mathrm{III}), \mathrm{Co}(\mathrm{II}), \mathrm{Ni}$ (II) to form PAEI hydrogels. By the treatment with salts of $\mathrm{Co}$ (II) or $\mathrm{Ni}(\mathrm{II})$, stable hydrogels were not obtained. On the other hand, Fe(II) and $\mathrm{Ru}$ (III) gave stable hydrogels $(\mathbf{9 a}, 9 \mathbf{9})$ through the coordination of the metal cation to the bipyridyl pendants. Needless to say, large stability constants for the complexation are required to form stable gels. Moreover, the kinetic inertness of the resulting gels seems to play an important role for the stability of the network.

The inertness and the stability constants of the bipyridyl-metal ion complex were affected by the reaction conditions such as $\mathrm{pH}$, temperature and solvent. This means that the stability of the coordination gel can be con- trolled by these reaction media. As a preliminary example, Fe(II)-bipyridyl-modified PAEI gels (9a) were stable in water at room temperature for a few days, but were redissolved in refluxing ethanol within an hour. The resulting red solution was concentrated slowly to the dry state under atmospheric pressure to give a polyoxazoline hydrogel again in a reversible manner. This interconversion from hydrogel to soluble polymer by heating can be regarded as a novel thermally reversible hydrogel system achieved by means of the kinetic control of the ligand exchange reaction. The reversible interconversion system, including the above thermal reversible system by the control of the thermodynamic stability and/or the kinetic inertness, will be discussed in a forthcoming paper.

\section{REFERENCES AND NOTES}

1. W. Kuhn, I. Toth, and H. J. Kuhn, Makromol. Chem., 60, 77 (1963).

2. H. Yokoi, S. Kawata, and M. Iwaizumi, J. Am. Chem. Soc., 108, 3361 (1986) and references therein.

3. H. R. Allcock and S. Kwon, Macromolecules, 22, 75 (1989).

4. E. Pezron, A. Ricard, F. Lafuma, and R. Audebert, Macromolecules, 21, 1121 (1988).

5. E. Pezron, L. Leibler, A. Ricard, and R. Audebert, Macromolecules, 21, 1126 (1988).

6. J. A. N. Zasadzinski, A. Chu, and R. K. Prud'homme, Macromolecules, 19, 2960 (1986).

7. K. Kamide, K. Yasuda, and K. Okajima, Polym. J., 20, 259 (1988).

8. C. Allain and L. Salom, Macromolecules, 20, 2958 (1987).

9. J. W. Rosthauser and A. Winston, Macromolecules, 14, 538 (1981).

10. D. Braun and H. Boudevska, Eur. Polym. J., 12, 525 (1976).

11. For example, H. Nishide, J. Deguchi, and E. Tsuchida, Chem. Lett., 169 (1976).

12. For example, C. Lecat-Tillier, F. Lafuma, and C. Quivoron, Eur. Polym. J., 16, 437 (1980).

13. H. C. Yen, B. E. Eichinger, and N. H. Andersen, $J$. Polym. Sci., Polym. Chem. Ed., 20, 2575 (1982).

14. N. Iwamoto, B. E. Eichinger, and N. H. Andersen, Rubber Chem. Technol., 57, 944 (1984).

15. S. Kopolow, T. E. Hgen Esch, and J. Smid, Macromolecules, 6, 133 (1973).

16. A. R. Samarakandy, N. Chatterjee, and B. E. 
Eichinger, Am. Chem. Soc., Div. Polym. Chem., 26(2), 50 (1980).

17. Y. Hirose, T. Amiya, Y. Hirokawa, and T. Tanaka, Macromolecules, 20, 1342 (1987) and references cited therein.

18. Y. Gnanou, G. Hild, and P. Rempp, Macromolecules., 17, 945 (1984).

19. J. Baselga, M. A. Llorente, J. L. Nieto, I. Hernandez-Fuentes, and I. F. Pierola, Eur. Polym. $J ., 25,477$ (1989) and references cited therein.

20. Reviews of the polymerization of cyclic imino ethers: (a) S. Kobayashi and T. Saegusa, in "Ring-Opening Polymerization," Vol. 2, K. J. Ivin and T. Saegusa, Ed., Elsevier Applied Science Publishers, Essex, U.K., 1984, p 761. (b) S. Kobayashi and T. Saegusa, Makromol. Chem., Suppl., 12, 11 (1985). (c) T. Saegusa, Makromol. Chem., Macromol., Symp., 13/14, 111 (1988). (d) T. Saegusa and Y. Chujo, in "Frontiers of Macromolecular Science," Blackwell Scientific Publications, 1989, p 119.

21. S. Kobayashi, M. Kaku, and T. Saegusa, Macromolecules, 21, 334 (1988).

22. Y. Chujo, Y. Yoshifuji, K. Sada, and T. Saegusa, Macromolecules, 22, 1074 (1989).

23. Y. Chujo, K. Sada, K. Matsumoto, and T. Saegusa, Macromolecules, 23, 1234 (1990).

24. Y. Chujo, K. Sada, and T. Saegusa, Macromolecules, 23, 2693 (1990).

25. Y. Chujo, K. Sada, and T. Saegusa, Macromolecules,
23, 2636 (1990).

26. W. R. McWhinnie and J. D. Miller, Adv. Inorg. Chem. Radiochem., 12, 135 (1969).

27. A. Juris, F. Barigelletti, S. Camagna, V. Balzani, P. Belser, A. von Zelewsky, Coord. Chem. Rev., 84, 85 (1988).

28. T. J. Meyer, Acc. Chem. Res., 22, 163 (1989).

29. J. M. Lehn, Angew. Chem. Int. Ed. Engl., 27, 89 (1988) and references cited therein.

30. Recent papers for polymeric ruthenium bipyridyl complexes; (a) J. L. Bourdelande, C. Campà, J. Font, and P. De March, Eur. Polym. J., 25, 197 (1989). (b) J. N. Younathan, S. F. McClanahan, and T. J. Meyer, Macromolecules, 22, 1048 (1989) and references cited therein.

31. K. Hanafusa, J.-I. Higashi, T. Koyama, H. Shirai, N. Hojo, and A. Kurose, Makromol. Chem., 190, 1 (1989).

32. L. Della Ciana, I. Hamachi, and T. J. Meyer, J. Org. Chem., 54, 1731 (1989).

33. R. M. Smith, A. E. Martell, Ed., "Critical Stability Constant," Vol. 2, Plenum Press, New York, N.Y., 1975, p 235.

34. F. Basolo and R. G. Pearson, "Mechanisms of Inorganic Reactions," 2nd ed, Wiley, New York, N.Y., 1967, Chapter 3, and see also, F. Basolo, J. C. Hayes, and H. M. Neumann, J. Am. Chem. Soc., 76, 3807 (1954).

35. Similar results were obtained, see ref 22,24 , and 25 . 